University of Wollongong

Research Online

Australian Institute for Innovative Materials -

Papers

Australian Institute for Innovative Materials

$1-1-2014$

Complementary terahertz absorption and inelastic neutron study of the dynamic anisotropy contribution to zone-center spin waves in a canted antiferromagnet $\mathrm{NdFeO3}$

\author{
Evan Constable \\ University of Wollongong, evanc@uow.edu.au \\ David L. Cortie \\ University of Wollongong, dlc422@uowmail.edu.au \\ Josip Horvat \\ University of Wollongong, jhorvat@uow.edu.au \\ Roger A. Lewis \\ University of Wollongong, roger@uow.edu.au \\ Zhenxiang Cheng \\ University of Wollongong, cheng@uow.edu.au
}

See next page for additional authors

Follow this and additional works at: https://ro.uow.edu.au/aiimpapers

Part of the Engineering Commons, and the Physical Sciences and Mathematics Commons

Research Online is the open access institutional repository for the University of Wollongong. For further information contact the UOW Library: research-pubs@uow.edu.au 


\title{
Complementary terahertz absorption and inelastic neutron study of the dynamic anisotropy contribution to zone-center spin waves in a canted antiferromagnet $\mathrm{NdFeO3}$
}

\begin{abstract}
We employ a combination of pulsed- and continuous-wave polarized terahertz spectroscopy techniques to probe temperature-dependent spin waves in the antiferromagnet NdFeO3. Our optical data span 1.6-467 $\mathrm{K}$ and reveal a conspicuous spin reorientation between 110 and $170 \mathrm{~K}$, during which the lowerenergy mode softens completely. Complementary inelastic neutron scattering reveals that the frequencies of the optically excited spin waves are consistent with a temperature-variable spin gap in the low-energy spin-wave dispersion of $\mathrm{NdFeO3.} \mathrm{The} \mathrm{result} \mathrm{links} \mathrm{the} \mathrm{temperature} \mathrm{dependence} \mathrm{of} \mathrm{the} \mathrm{spin}$ waves to a dynamic in-plane anisotropy. The magnetic anisotropy is calculated based on the results of the optical measurements. The change observed in the anisotropy energy along the a and c crystal axes suggests that the spin reorientation evident in $\mathrm{NdFeO3}$ is driven by temperature-dependent in-plane anisotropy.
\end{abstract}

\section{Keywords}

contribution, zone, center, spin, waves, canted, antiferromagnet, ndfeo3, absorption, inelastic, neutron, complementary, study, terahertz, dynamic, anisotropy

Disciplines

Engineering | Physical Sciences and Mathematics

\section{Publication Details}

Constable, E., Cortie, D. L., Horvat, J., Lewis, R. A., Cheng, Z., Deng, G., Cao, S., Yuan, S. \& Ma, G. (2014). Complementary terahertz absorption and inelastic neutron study of the dynamic anisotropy contribution to zone-center spin waves in a canted antiferromagnet NdFe03. Physical Review B: Condensed Matter and Materials Physics, 90 054413-1-054413-9.

\section{Authors}

Evan Constable, David L. Cortie, Josip Horvat, Roger A. Lewis, Zhenxiang Cheng, Guochu Deng, Shixun Cao, Shujuan Yuan, and Guohong Ma 


\title{
Complementary terahertz absorption and inelastic neutron study of the dynamic anisotropy contribution to zone-center spin waves in a canted antiferromagnet $\mathrm{NdFeO}_{3}$
}

\author{
Evan Constable, ${ }^{1,2,{ }^{*}}$ D. L. Cortie,,${ }^{1,2,3}$ Joseph Horvat, ${ }^{1,2}$ R. A. Lewis, ${ }^{1,2}$ Zhenxiang Cheng, ${ }^{1}$ Guochu Deng, ${ }^{3}$ \\ Shixun Cao, ${ }^{4}$ Shujuan Yuan, ${ }^{4}$ and Guohong $\mathrm{Ma}^{4}$ \\ ${ }^{1}$ Institute for Superconducting and Electronic Materials, University of Wollongong, Wollongong, New South Wales 2522, Australia \\ ${ }^{2}$ School of Physics, University of Wollongong, New South Wales 2522, Australia \\ ${ }^{3}$ Bragg Institute, Australian Nuclear Science and Technology Organisation, New Illawarra Road, Lucas Heights, \\ New South Wales 2233, Australia \\ ${ }^{4}$ Department of Physics, Shanghai University, Shanghai 200444, People's Republic of China \\ (Received 24 May 2014; revised manuscript received 27 July 2014; published 19 August 2014)
}

\begin{abstract}
We employ a combination of pulsed- and continuous-wave polarized terahertz spectroscopy techniques to probe temperature-dependent spin waves in the antiferromagnet $\mathrm{NdFeO}_{3}$. Our optical data span $1.6-467 \mathrm{~K}$ and reveal a conspicuous spin reorientation between 110 and $170 \mathrm{~K}$, during which the lower-energy mode softens completely. Complementary inelastic neutron scattering reveals that the frequencies of the optically excited spin waves are consistent with a temperature-variable spin gap in the low-energy spin-wave dispersion of $\mathrm{NdFeO}_{3}$. The result links the temperature dependence of the spin waves to a dynamic in-plane anisotropy. The magnetic anisotropy is calculated based on the results of the optical measurements. The change observed in the anisotropy energy along the $a$ and $c$ crystal axes suggests that the spin reorientation evident in $\mathrm{NdFeO}_{3}$ is driven by temperature-dependent in-plane anisotropy.
\end{abstract}

DOI: 10.1103/PhysRevB.90.054413

PACS number(s): 76.50.+g, 75.30.Gw, 78.20.Ls, 78.70.Nx

\section{INTRODUCTION}

The optical excitation and control of spins in magnetic materials promises new avenues for devices that couple photonics and spintronic functionality, with the prospects of a new paradigm in information processing [1]. An understanding of the behavior of the magnetic interactions in candidate materials is essential to the design of devices that exhibit such properties. The interaction of terahertz $(\mathrm{THz})$ frequency electromagnetic radiation with antiferromagnetic materials is particularly appealing as it offers the possibility of monitoring ultrafast magnetization processes in the time domain [2] as well as inducing magnetization switching $[3,4]$. Furthermore, the $\mathrm{THz}$ energy range is comparable with the low-energy terms of the magnetic Hamiltonian such as magnetic anisotropy and the Dzyaloshinsky-Moriya (DM) interaction, providing a route to probe these directly. This is important in the development of materials suitable for spintronic applications [5], but typically is challenging for other experimental techniques such as inelastic neutron scattering [6,7], and even for computational methods such as density functional theory [8]. Recent experiments using $\mathrm{THz}$ time domain spectroscopy (TDS) with ultrafast pulses have shown characteristic signals which were interpreted as the pumping and probing of antiferromagnetic magnetic spin-wave modes on femtosecond time scales [9-11]. New mechanisms such as induced precession and free-induction decay have been proposed which, if accurate, promise new avenues to optically address specific spins and control spin diffusion.

Absorption by spin-wave excitation may occur when the time-dependent magnetic field, $\mathbf{H}$, of a $\mathrm{THz}$ wave, couples to $k=0$ spin-wave oscillations through a Zeeman torque

\footnotetext{
*ec028@uowmail.edu.au
}

on the electron's spin moment $\mathbf{T}=\gamma \mathbf{S} \times \mathbf{H}$. However, due to the electromagnetic nature of $\mathrm{THz}$ waves it is difficult to differentiate a magnetic excitation from an electrical one based solely on the optical spectrum, since the time-dependent electrical field, E, may also couple to dipolar moments within the material [12]. Inelastic neutron scattering (INS) may identify magnetic excitations through its unique ability to collect scattered spectra at specific locations in the reciprocal space of the magnetic lattice [7]. Careful monitoring of the form and structure factor of excitations can confirm their magnetic origin. Thus, INS is a strong complementary technique to $\mathrm{THz}$ spectroscopy.

Here we combine pulsed- and continuous-wave $\mathrm{THz}$ spectroscopy techniques as well as INS to probe spin-wave excitations in antiferromagnetically ordered neodymium orthoferrite $\left(\mathrm{NdFeO}_{3}\right)$. The results obtained through optical methods establish the polarization sensitivity of the spin excitations and reveal a spin reorientation (SR) of the $\mathrm{Fe}-\mathrm{Fe}$ spin sublattice over 110-170 K. Comparison with INS data suggests that there is a relation between a spin-wave gap that develops at low temperature and the SR. This supports the presence of a temperature-variable magnetic anisotropy in the Hamiltonian, as previously proposed for this class of system $[13,14]$. The results are used to calculate this anisotropy. The ability to directly measure the effective magnetic anisotropy of an antiferromagnet and its temperature dependence is novel and may help to resolve conceptual difficulties and debates in the literature, for example, the role anisotropy $[13,14]$ and magnetic exchange bias [15] play in the SR phenomena of rare-earth orthoferrites.

The paper is structured as follows: In Sec. II we present a simplified Hamiltonian to model the Fe-Fe spin sublattice and show two optically-active acoustic spin waves as solutions to the model. The experimental details of the measurements are then outlined. In Sec. III we present the results. We first use $\mathrm{THz}$ spectroscopy to identify the two modes and demonstrate 
their orthogonality. Temperature-varied measurements of the spin waves are also presented which reveal a strong temperature dependence on mode frequency as well as a rotation of the spin-lattice state. Next, we present the results of INS experiments. These are used to confirm the magnetic origin of the modes. A spin gap associated with magnetic anisotropy is also observed. Finally, the temperature-dependent results are combined and used to extract changes in the effective anisotropy over the temperature range studied. This is followed by a brief discussion on the significance of the results and how they may relate to the SR phenomenon. Section IV concludes the paper.

\section{METHODS}

\section{A. Material overview}

$\mathrm{NdFeO}_{3}$ is a rare-earth orthoferrite $\left(\mathrm{RFeO}_{3}\right)$ perovskite of the $D_{2 h}^{16}(P b n m)$ space group [16]. The nearest-neighbor $\mathrm{Fe}^{3+}$ ions in the $\mathrm{RFeO}_{3}$ series are antiferromagnetically ordered through a $\mathrm{Fe}-\mathrm{O}-\mathrm{Fe}$ superexchange at temperatures below $\sim 700 \mathrm{~K}$ [17]. A weak ferromagnetic moment perpendicular to the antiferromagnetic ordering axis occurs due to subtle spin canting as a result of the antisymmetric DM interaction [18]. At room temperature, for $\mathrm{NdFeO}_{3}$, the $\mathrm{Fe}^{3+}$ spin state equates to antiferromagnteic ordering along the $a$ crystal axis with the ferromagnetic moment directed along the $c$ axis, or $G_{x} F_{z}$ in the notation of Bertaut where the magnetic axes $x, y$, and $z$ are aligned with the crystallographic axes $a, b$, and $c$, respectively $[19,20]$. The $\mathrm{RFeO}_{3}$ series is known to exhibit a SR transition involving a continuous and coherent temperature-driven rotation of the ordered spin lattice through $90^{\circ}$ from one principal axis to another [21]. For $\mathrm{NdFeO}_{3}$ this involves the transition $G_{x} F_{z} \rightarrow G_{z} F_{x}$ where below the SR temperature the spin state is antiferromagnetically aligned along the $c$ axis with the ferromagnetic moment along the $a$ axis.

In general, a four sublattice Hamiltonian is required to describe the $\mathrm{Fe}^{3+}$ spin system in $\mathrm{NdFeO}_{3}$ due to the four inequivalent $\mathrm{Fe}^{3+}$ sites [22]. However, it has been shown that a simplified two sublattice Hamiltonian is an adequate approximation for the low-energy interactions associated with acoustic spin waves [23]. The Hamiltonian may therefore be constructed with a single isotropic exchange interaction coupling nearest-neighbor $\mathrm{Fe}^{3+}$ ions and an antisymmetric $\mathrm{DM}$ interaction which is a consequence of the $\mathrm{Fe}-\mathrm{O}-\mathrm{Fe}$ superexchange spin coupling mechanism [23]. An effective second-order anisotropy is included to stabilize the spin sublattices with respect to the crystal lattice. The effective anisotropy is chosen to represent the contribution of all the microscopic anisotropy subterms in the Hamiltonian. The Hamiltonian is then [24]

$$
H=2 J \sum_{i, j} \mathbf{S}_{i} \cdot \mathbf{S}_{j}+\sum_{i, j}\left(\mathbf{D} \cdot \mathbf{S}_{i} \times \mathbf{S}_{j}\right)+\sum_{i, j}\left(\mathbf{K}_{\mathrm{eff}} \cdot \mathbf{S}_{i}\right)^{2} .
$$

Here $J\left(0.66 \mathrm{THz} / 22 \mathrm{~cm}^{-1} / 2.7 \mathrm{meV}\right)$ and $D(0.028 \mathrm{THz} / 0.95$ $\left.\mathrm{cm}^{-1} / 0.1 \mathrm{meV}\right)$ are the isotropic Heisenberg and antisymmetric DM exchange constants, $S(=5 / 2)$ is the spin moment of the $i$ th and $j$ th nearest-neighbor $\mathrm{Fe}^{3+}$ ions, and $\mathbf{K}_{\text {eff }}$ is the effective anisotropy [29]. Following the standard procedure, we set the magnetic axes along the crystallographic axes $(x \| a$, $y\|b, z\| c)$. At room temperature in the $G_{x} F_{z}$ state, $\mathbf{D}=D \hat{z}$ and the second term becomes $D \sum_{n n}\left(S_{i}^{z} S_{j}^{x}-S_{i}^{x} S_{j}^{z}\right)$. Neutron diffraction $[16,20]$ and magnetometry $[25,26]$ studies indicate that the rotation of the spin moments is confined to the $a c$ plane. Therefore, we set $\mathbf{K}_{\mathrm{eff}}=\left(K_{x}, 0, K_{z}\right)$ and the last term becomes $K_{x}\left[\sum_{i}\left(S_{i}^{x}\right)^{2}+\sum_{j}\left(S_{j}^{x}\right)^{2}\right]+K_{z}\left[\sum_{i}\left(S_{i}^{z}\right)^{2}+\sum_{j}\left(S_{j}^{z}\right)^{2}\right]$, where $K_{x}$ and $K_{z}$ are the second-order anisotropic field energies along the $a$ and $c$ crystal axes. Magnetic interactions of the $\mathrm{Nd}^{3+}$ ions are neglected over the temperature range used, given the low ordering temperature $(\sim 1.5 \mathrm{~K})$. The time-dependent spin waves are then governed by $J, D, K_{x}$, and $K_{z}$. The solutions for the $k=0$ modes of Eq. (1) are

$$
\begin{aligned}
\hbar \omega_{\mathrm{F}} & =\left\{24 J S\left[2\left(K_{x}-K_{z}\right) S\right]\right\}^{1 / 2} \text { and } \\
\hbar \omega_{\mathrm{AF}} & =\left\{24 J S\left[6 D S \tan \beta+2 K_{x} S\right]\right\}^{1 / 2},
\end{aligned}
$$

where $\beta(8.5 \mathrm{mrad})$ is the antiferromagnetic canting angle between the $\mathrm{Fe}^{3+}$ magnetic moments of each spin sublattice $[23,24]$. The two modes are displayed in Figs. 1(a) and 1(c) [23], respectively.
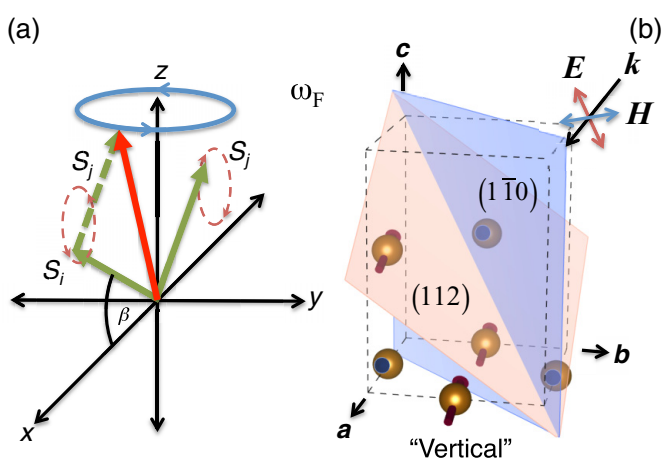

(c)
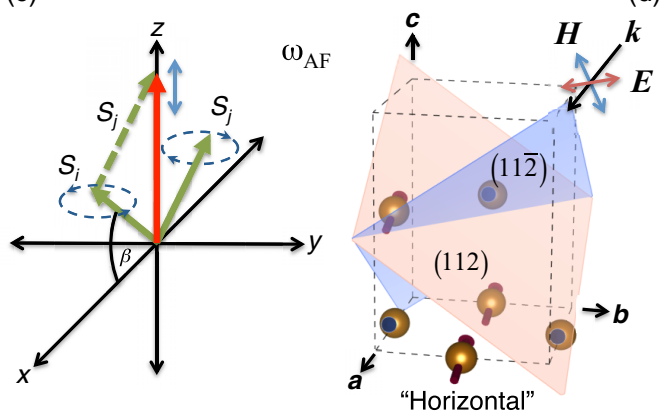

FIG. 1. (Color online) Optically active spin-wave modes in $\mathrm{NdFeO}_{3}$ and their excitation planes. (a) $\omega_{\mathrm{F}}$ combines in-phase oscillations of the antiparallel spin components $\left(S_{i}\right.$ and $\left.S_{j}\right)$ along the $x$ and $y$ axes with out-of-phase oscillations of the $z$ components. The net effect is a low-frequency rotation of the weak ferromagnetic moment around the $z$ axis. (b) Fe-Fe magnetic sublattices (at room temperature) sectioned along the (112) plane (pink surface), the sample face. Incident radiation polarized within (110) (blue surface) excites the $\mathrm{F}$ mode. (c) $\omega_{\mathrm{AF}}$ has out-of-phase oscillations of the antiparallel $x$ and $y$ components and in-phase oscillations of the $z$ components. This gives a net magnetic oscillation along the $z$ axis. (d) Incident radiation polarized within (112) excites the AF mode. 
Using linearly polarized light each mode can be excited separately, since the optically active components of the modes are in the $x y$ plane for $\omega_{\mathrm{F}}$ and along the $z$ axis for $\omega_{\mathrm{AF}}$. As Fig. 1 shows, $\omega_{\mathrm{F}}$, referred to as the $\mathrm{F}$ mode, features a pseudoexcitation of the ferromagnetic moment. Given the rocking motion of the antiparallel spin moments in the $x z$ plane, the F-mode energy depends on the difference in the anisotropic fields $\left(K_{x}-K_{z}\right)$. On the other hand, $\omega_{\mathrm{AF}}$, referred to as the AF mode, features a twisting motion about the antiferromagnetic axis. Its energy is sensitive to both the forces along the antiferromagnetic axis $\left(K_{x}\right)$ and to the amount of spin canting $(D \tan \beta)$.

Figure 1 also demonstrates how we conducted the optical experiments on a (112)-cut single crystal with a polarized (E) incident beam. As in Fig. 1(b), when $\mathbf{E}$ is confined to the (1110) plane, $\mathbf{H}$ is in the (11) $)$ plane. At normal incidence to (112), $\mathbf{H}$ oscillates exclusively in the $x y$ plane, with the $x$ and $y$ components out of phase by $\pi / 2$. The optically active $x$ and $y$ components of the spin moments have the same time-dependent relationship and the $\omega_{\mathrm{F}}$ mode is excited [Fig. 1(a)]. With $\mathbf{H}$ lacking a $z$ component, $\omega_{\mathrm{AF}}$ is not excited. A $90^{\circ}$ rotation in polarization puts $\mathbf{E}$ in $(11 \overline{2})$ and $\mathbf{H}$ in (11) At normal incidence to (112), $\mathbf{H}$ has components along all three axes. The $z$ component of $\mathbf{H}$ couples to the oscillating magnetic dipole of the $\omega_{\mathrm{AF}}$ mode, which is now excited. At the same time, in the $x y$ plane the oscillations of the $x$ and $y$ components of $\mathbf{H}$ are in phase and this cancels the $\omega_{\mathrm{F}}$ mode. A (112)-cut crystal is thus ideal for measurement of the temperature dependence of the two modes, as each mode can be exclusively tracked through the SR using a single sample. For simplicity in describing the orthogonal polarizations later, we call the polarization of $\mathbf{E}$ confined to the (110) plane "vertical" and $\mathbf{E}$ confined to the (112) plane "horizontal." To assist in identifying the orientations in the figures, data shown in red is for the vertical polarization, while data shown in blue is for the horizontal polarization.

\section{B. Experimental details}

A single crystal of $\mathrm{NdFeO}_{3}$ was grown by the floating zone method [26]. A disk (7 mm diameter, $1.95 \mathrm{~mm}$ thick) cut from the as-grown $\mathrm{NdFeO}_{3}$ single crystal boule was used for optical experiments and a larger disk $(7 \mathrm{~mm}$ diameter, $5 \mathrm{~mm}$ thick) from the same boule was used for INS experiments. Optical measurements from 300 to $470 \mathrm{~K}$ in the spectral range $0.1-2 \mathrm{THz}$ were performed using a Zomega $\mathrm{THz}$ time domain spectrometer. Scans were taken with a maximum time delay of $66 \mathrm{ps}$, producing a spectral resolution of $0.015 \mathrm{THz}$ $\left(0.5 \mathrm{~cm}^{-1} / 0.06 \mathrm{meV}\right)$. The sample was mounted on a heated aluminum block with a $5 \mathrm{~mm}$ aperture to allow transmission measurements. The temperature was controlled by adjusting the current to the heating element [27]. Measurements from 1.6 to $300 \mathrm{~K}$ in the spectral range $0.3-2 \mathrm{THz}$ utilized a modified Polytec FIR 25 Fourier transform interferometer interfaced to the low-temperature insert of a He cooled 7-T Oxford split-coil superconducting magnet. Optical path lengths of $2 \mathrm{~cm}$ were used giving an unapodized spectral resolution of $0.5 \mathrm{~cm}^{-1}(0.015 \mathrm{THz} / 0.06 \mathrm{meV})$. Measurements in the spectral range $0.08-0.3 \mathrm{THz}$ from 40 to $300 \mathrm{~K}$ were made using a continuous-wave photomixing $\mathrm{THz}$ spectrometer interfaced to the same cryostat [28]. The photomixing spectrometer is a tunable quasimonochromatic source and data are taken directly in the frequency domain at a resolution of $0.001 \mathrm{THz}$ $\left(0.03 \mathrm{~cm}^{-1} / 4 \mu \mathrm{eV}\right)$. In all cases optical measurements were made in transmission geometry, with the incident radiation normal to the (112) crystal face. The INS experiments were carried out on the Australian OPAL reactor TAIPAN triple-axis spectrometer at constant $E_{\mathrm{f}}(\simeq 14.87 \mathrm{meV})$ with a resolution of $0.25 \mathrm{meV}\left(0.06 \mathrm{THz} / 2 \mathrm{~cm}^{-1}\right)$. Constant $Q$ energy scans from 0 to $15 \mathrm{meV}$ were made at the antiferromagnetic zone center in the $a c$ and $b c$ planes at 11 temperature steps between 3 and 298 K. Constant energy $Q$ scans were also performed at 175 , 50,25 , and $3 \mathrm{~K}$, around the magnetic zone center $\mathbf{Q}=(h 01)$.

\section{RESULTS AND DISCUSSION}

\section{A. Terahertz spectroscopy}

TDS of $\mathrm{NdFeO}_{3}$ at room temperature $(300 \mathrm{~K})$, with $\mathbf{E}$ in both the horizontal and vertical polarizations, is shown in Fig. 2(a). As represented in Fig. 1, the two spin waves should

(a)
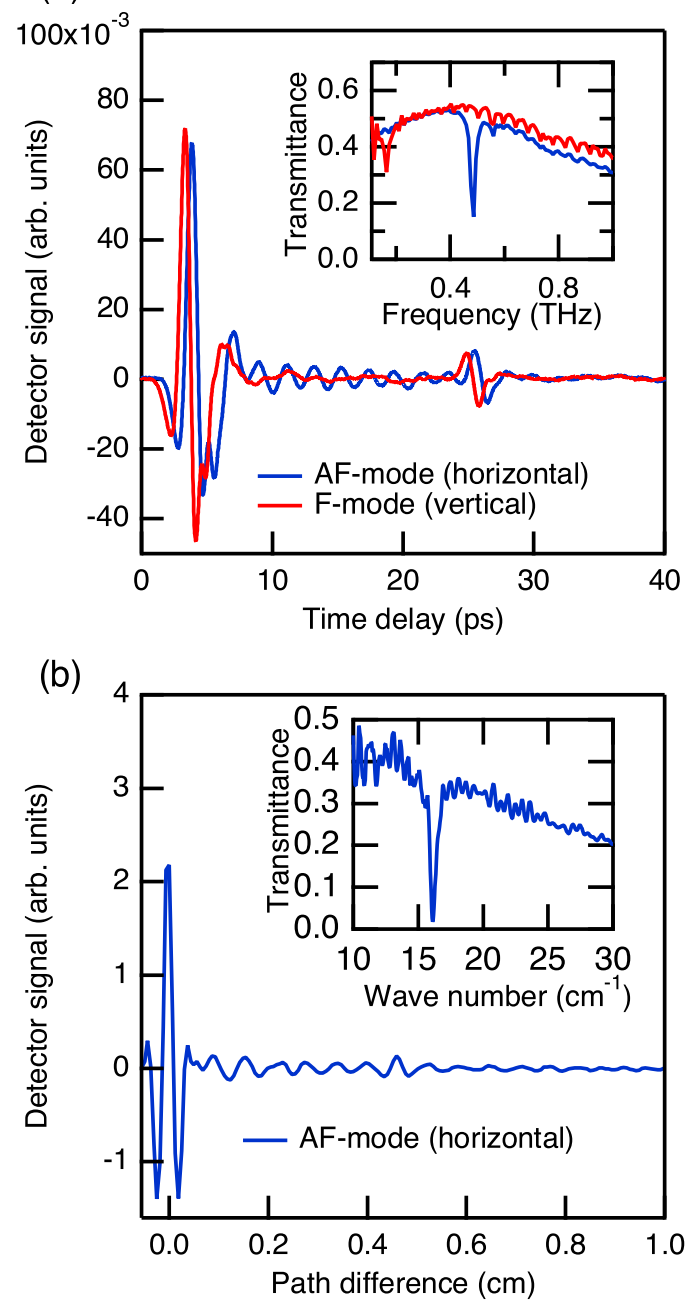

FIG. 2. (Color online) (a) $\mathrm{THz} \mathrm{TD}$ spectra of $\mathrm{NdFeO}_{3}$ at $300 \mathrm{~K}$ and (b) far-infrared interferogram at $295 \mathrm{~K}$ with transmittance (insets) calculated by the ratio of the Fourier transformed spectrum to a reference spectrum. 
have orthogonal excitation axes and therefore appear exclusively in the horizontal and vertical polarization orientations. This is evident by the periodic oscillations in the spectra, which indicate absorption due to resonance of the F and AF modes.

The period of the oscillation in the horizontal polarization is $\sim 2.06$ ps while the period of the much weaker oscillation in the vertical polarization is $\sim 6.06 \mathrm{ps}$. As seen in the inset, these correspond to resonances at 0.485 and $0.165 \mathrm{THz}$. No mixing of the modes in each polarization is observed and the frequencies agree with those of spin waves in $\mathrm{NdFeO}_{3}$ excited by Raman scattering [29].

The interferogram of Fig. 2(b) demonstrates that the oscillation observed in the TDS spectra [Fig. 2(a)] is not unique to time-resolved measurements. The same AF mode is observed using conventional far-infrared Michelson interferometry. The wavelength of the oscillation is $\sim 0.0618 \mathrm{~cm}$, which is equivalent to a resonance at $16.17 \mathrm{~cm}^{-1}$, or $0.485 \mathrm{THz}$. The softening in energy compared to the measurements shown in Fig. 2(a) are due to the change in temperature from 300 to $290 \mathrm{~K}$. (The F mode could not be observed at this temperature as its low frequency falls below the spectral bandwidth of the interferometer.) To observe both modes clearly by the interferometer, the sample must be $<40 \mathrm{~K}$ (so the F-mode frequency hardens to above $8 \mathrm{~cm}^{-1}$ ). Relative to room temperature, the two modes should flip in polarization due to the $90^{\circ} \mathrm{SR}$, and shift in frequency due to changes in the internal anisotropic fields. An interferogram for each polarization at $7.5 \mathrm{~K}$ is shown in Fig. 3(a).

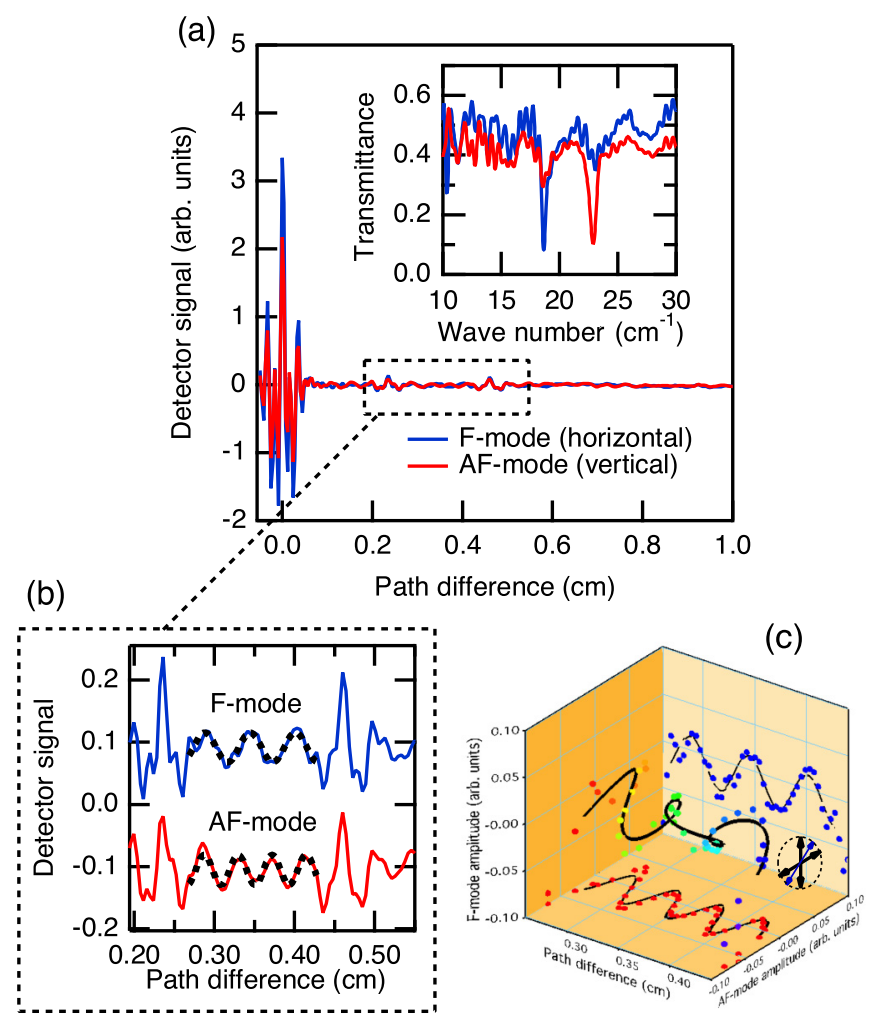

FIG. 3. (Color online) (a) Interferogram in both horizontal and vertical polarizations and equivalent transmittance spectra (inset) at 7.5 K. (b) Extracted section from (a) showing mode oscillation. The two traces are offset for clarity. (c) Visualization of mixed modes for off-axis polarization.
Figure 3(b) is a zoomed-in section extracted from the full interferogram demonstrating the mode oscillation. Here, we see that the wavelength of the oscillation for the AF mode is now $0.0435 \mathrm{~cm}$, equivalent to a resonance at $23 \mathrm{~cm}^{-1}$. The F-mode oscillation wavelength is $0.0538 \mathrm{~cm}$ giving a resonance at $18.6 \mathrm{~cm}^{-1}$. As seen in the inset of Fig. 3(a) there is now a trace of the F mode in the vertical polarization, which should be sensitive to the AF mode only. On the other hand, there is no trace of the AF mode in the F-mode sensitive horizontal polarization. As is demonstrated in the mixed plot of the two modes [Fig. 3(c)], any polarization orientation away from either vertical or horizontal should couple to both modes. Therefore, if the leaking of the F mode in the vertical polarization was due to a SR other than $90^{\circ}$, we would expect to see both modes in each polarization. The same argument can discount any effects due to polarizer inefficiency. We therefore attribute this observation to the selection rules in the newly-oriented geometry at temperatures below the SR. In the $G_{z} F_{x}$ state the F-mode precesses around the $z$ axis. The horizontal polarization will couple to this mode. However, the vertical polarization will also have a projection into the $z y$ plane. Unlike in the $G_{x} F_{z}$ state at room temperature, here the H-field components do not fully cancel the F mode and its signature can be seen in the vertical polarization.

As previously mentioned, the resonant frequencies of the spin-wave modes are strongly temperature dependent and therefore can provide information regarding the underlying temperature-dependent physics of the Hamiltonian [Eq. (1)]. The results of temperature-dependent transmission measurements in each polarization are shown in Fig. 4. For vertical polarization [Fig. 4(a)], the F mode softens as the sample is cooled from high temperature. From 300 to $180 \mathrm{~K}$ no data are available as the $\mathrm{F}$ mode softens below the bandwidth of our spectrometers. From $160 \mathrm{~K}$, as the SR begins, the AF mode strengthens in intensity as the spin sublattice rotates into alignment with the horizontal polarization.

On further cooling, the AF mode hardens considerably. In the horizontal polarization [Fig. 4(b)], the AF mode is observed at high temperatures. As the sample is cooled the AF mode first hardens, then softens and loses intensity during the SR transition, finally disappearing.

It is not until $\sim 40 \mathrm{~K}$ the $\mathrm{F}$ mode can be seen, as it hardens when the sample is cooled further. The peak intensity for the AF mode as a function of temperature is shown in Fig. 4(c). As the temperature is reduced and the thermal disruptions become weaker, the resonance modes strengthen.

As the temperature of the sample approaches the SR transition, the polarization sensitivity reveals the rotation through a weakening of the AF mode in this orientation due to a smaller projection of the spin moment along the excited axis. Correspondingly in the other orientation, the AF mode is seen to strengthen as the projection of the excited spin moment maximizes its alignment with the $\mathbf{H}$ field. The sensitivity to the alignment of the spin sublattice allows us to determine the temperatures $T_{1}=110 \mathrm{~K}$ and $T_{2}=170 \mathrm{~K}$ as the boundaries of the spin reorientation. A rapid weakening is also observed for our measurements at 1.6 and $1.8 \mathrm{~K}$. Presently, it is not clear whether this is due to a reduced spin moment projection in the plane of polarization (corresponding to another SR at lower temperatures), a reduction in the net magnetization of the $\mathrm{Fe}^{3+}$ 
(a) wave number $\left(\mathrm{cm}^{-1}\right)$

$\begin{array}{llllll}5 & 10 & 15 & 20 & 25 & 30\end{array}$

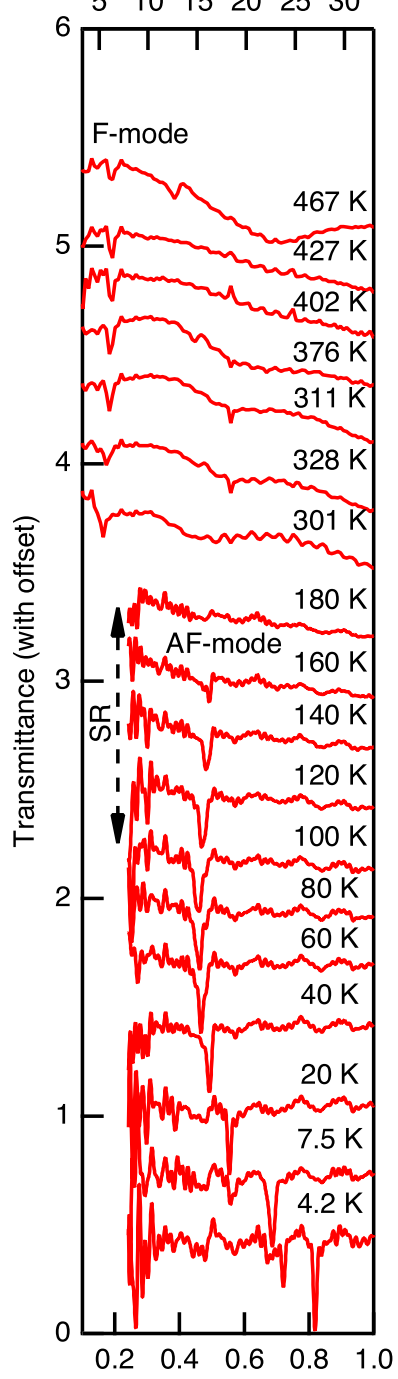

Frequency $(\mathrm{THz})$ (b) wave number $\left(\mathrm{cm}^{-1}\right)$

$\begin{array}{llllll}5 & 10 & 15 & 20 & 25 & 30\end{array}$

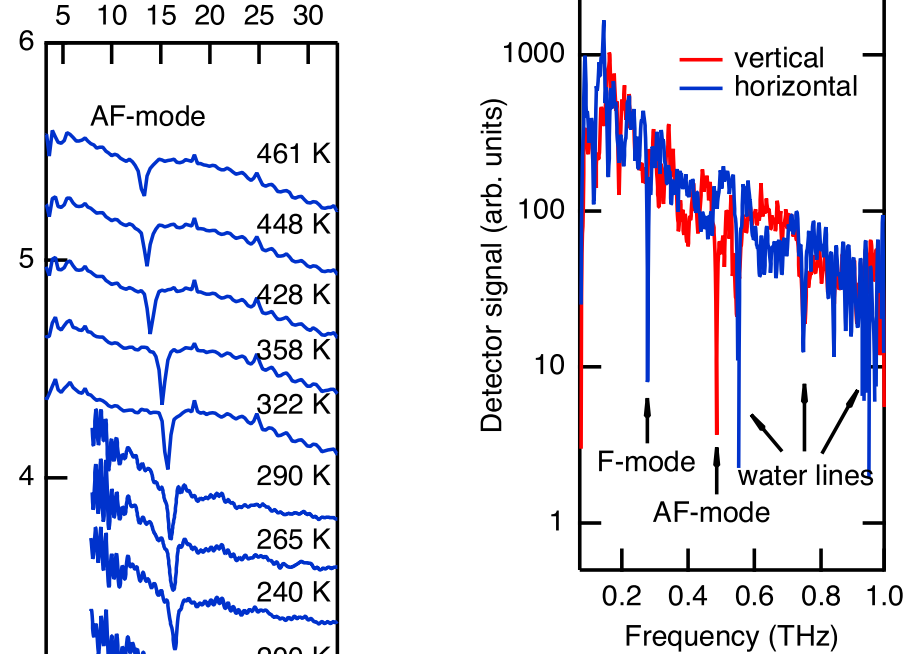

(b)

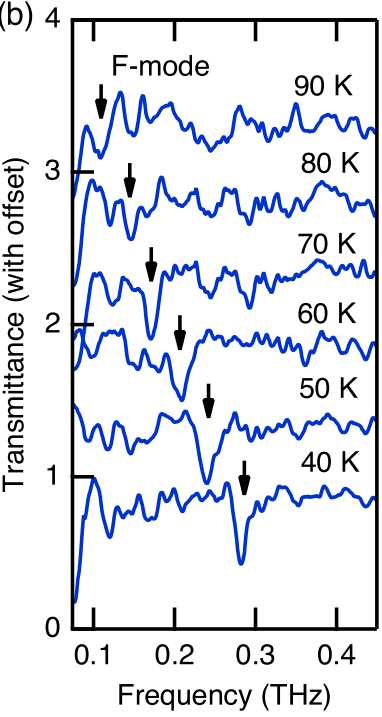

FIG. 5. (Color online) (a) Spectrum of $\mathrm{NdFeO}_{3}$ at $40 \mathrm{~K}$ taken using a quasimonochromatic photomixing $\mathrm{THz}$ spectrometer in the horizontal and vertical polarization orientations. Both the $\mathrm{F}$ mode and AF mode are observed at a high resolution of $1 \mathrm{GHz}$. Absorption lines at $0.55 \mathrm{THz}$ and above are due to water vapor. (b) Temperature dependence of $\mathrm{F}$ mode from 40 to $90 \mathrm{~K}$ showing mode softening.

sublattice, or simply a measurement artifact. Recent studies have indicated that exchange coupling between the $\mathrm{Nd}^{3+}$ ions may produce antiferromagnetic ordering in the $\mathrm{Nd}^{3+}$ sublattice at very low temperatures [20,30,31]. Competition between the corresponding $\mathrm{Fe}^{3+}$ and $\mathrm{Nd}^{3+}$ sublattice magnetic moments gives rise to a particularly unstable magnetic state where either of these scenarios is a possibility.

In the temperature-dependent anisotropy model for SR of Shane [13], $\left(K_{x}-K_{z}\right)$ is expected to reverse in sign over the SR transition. Therefore, one should observe complete softening of the F mode [Eq. (2)].

To observe softening of the F mode over 40-300 K, the quasimonochromatic photomixing $\mathrm{THz}$ spectrometer, boasting a lower-frequency limit and high resolution, was used. Representative spectra at $40 \mathrm{~K}$ are shown in Fig. 5(a). The $\mathrm{F}$ mode is at $0.28 \mathrm{THz}$ and the $\mathrm{AF}$ mode is at $0.485 \mathrm{THz}$. Interference fringes due to multiple reflections from the crystal face are filtered out by performing an inverse Fourier transform of the spectrum and attenuating the reflection peak in the inverse spectrum. The spectrum is then retransformed.

Transmittance spectra from 40-90 K are shown in Fig. 5(b). By $90 \mathrm{~K}$ the $\mathrm{F}$ mode was observed to weaken below our detection limits.

\section{B. Inelastic neutron scattering}

FIG. 4. (Color online) Temperature-dependent transmittance spectra of $\mathrm{NdFeO}_{3}$ across the SR. (a) Horizontal orientation. Data from 4.2 to $180 \mathrm{~K}$ were taken in an interferometer and truncated below $8 \mathrm{~cm}^{-1}$ due to noise. Data from 301 to $467 \mathrm{~K}$ are from a time domain spectroscopy system. (b) Vertical orientation; interferometer data from 4.2 to $290 \mathrm{~K}$; time domain data from 322 to $461 \mathrm{~K}$. (c) Temperature dependence of peak strength for AF mode. The SR is identified from 170 to $110 \mathrm{~K}$.

The results from a series of INS constant energy $\mathbf{Q}$ scans around the magnetic zone center $\mathbf{Q}=(101)$ and at several temperatures across the SR are shown in Fig. 6(a). Focus is given to energies less than $10 \mathrm{meV}$. The energies below $1.5 \mathrm{meV}$ are obscured by the elastic Bragg peak near the zone center due to the limited energy resolution of thermal neutrons. As the temperature is increased, the background signal is raised due to enhanced incoherent scattering. Despite 


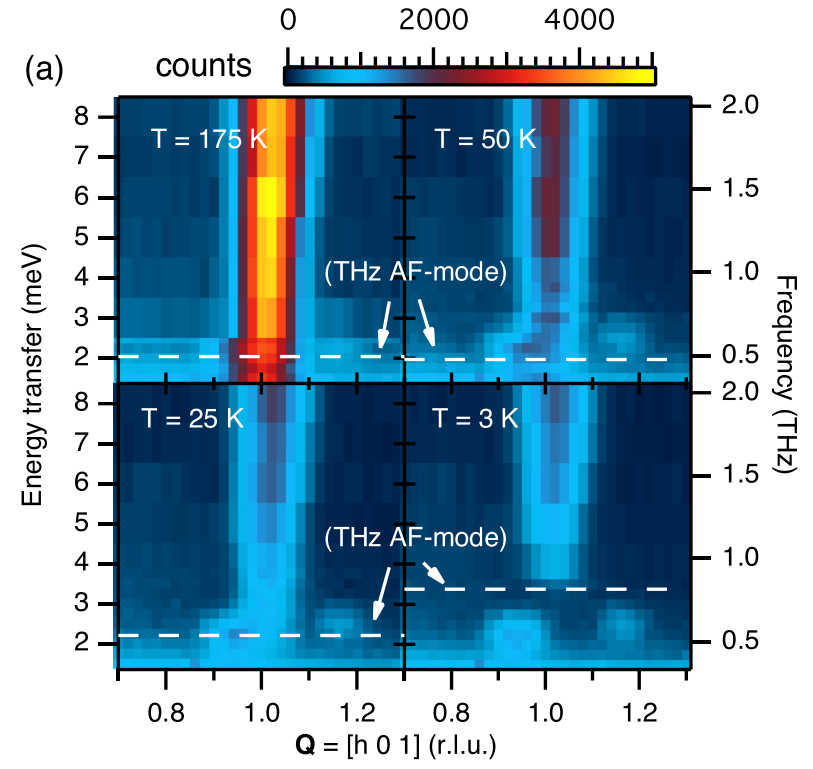

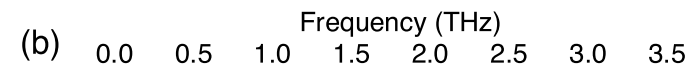

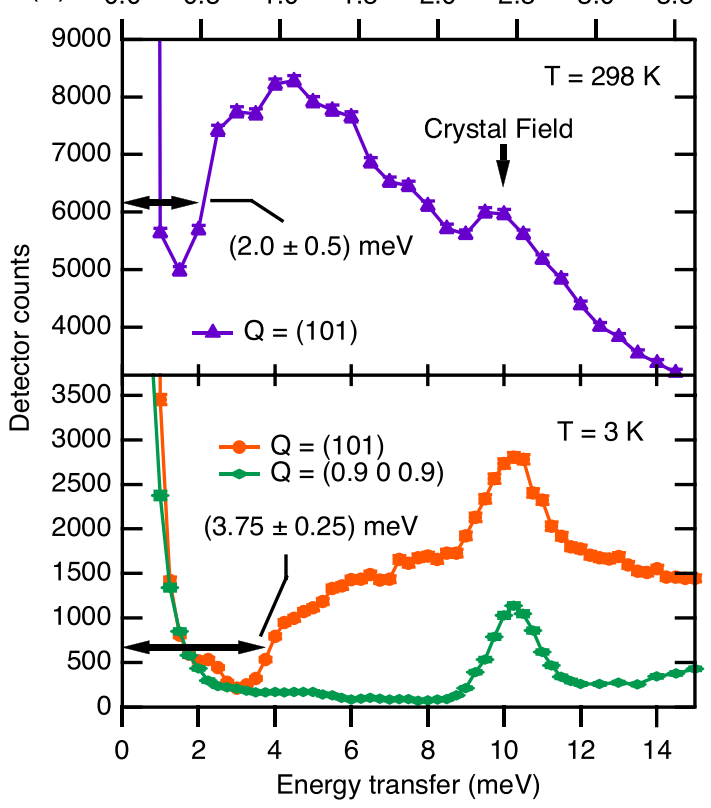

FIG. 6. (Color online) (a) Temperature evolution of low-energy spin-wave dispersion at the magnetic zone center $\mathbf{Q}=(101) . \mathbf{Q}=$ $(h k l)$ is given in reciprocal lattice units (r.l.u.). The dashed lines indicate the resonant frequency of the AF mode as measured by the optical $\mathrm{THz}$ techniques for the equivalent temperatures (the AF mode at $4.2 \mathrm{~K}$ is used for the $3 \mathrm{~K}$ dispersion plot). (b) Constant $\mathbf{Q}$ energy scans of the magnetic zone center $\mathbf{Q}=(101)$ at 298 and $3 \mathrm{~K}$.

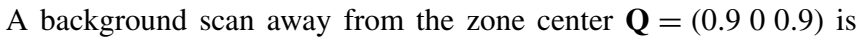
also shown in (b). Lines between data points are included as a guide for the eye. The error bars of the data points represent the standard deviation of the statistical neutron counts (in most cases the error bars are obscured by the markers).

these experimental limitations, the scattering experiments yield important information about the acoustic spin-wave dispersion.

At $175 \mathrm{~K}$, just above the $\mathrm{SR}$, the dispersion curve at the zone center mixes with the Bragg peak. As the sample is cooled a gap forms between the ground state and $k=0$ excited spin-wave state, most prominent in the data at $3 \mathrm{~K}$. In the standard isotropic Heisenberg antiferromagnet, acoustic spin waves have zero energy at the zone center. Their energies are lifted when in-plane anisotropies lower the magnetic symmetry [32]. The temperature development of a gapped dispersion therefore provides strong evidence for a temperature-dependent in-plane spin anisotropy and its rapid increase at temperatures below the SR suggests that the temperature-dependent anisotropy drives the SR. The development of two off-center peaks $\left[\mathbf{Q}=\left(\begin{array}{lll}0.95 & 0 & 1\end{array}\right)\right.$ and $\left.\left(\begin{array}{lll}1.2 & 0 & 1\end{array}\right)\right]$ with energies centered on $2.25 \mathrm{meV}$ at these temperatures is a peculiar result. The $\mathrm{Nd}^{3+}$ ions have not been seen to order for temperatures as low as $1.5 \mathrm{~K}[30]$, so it is unlikely that the peaks are due to coherent scattering of a newly-ordered state.

Since the peaks are separated from the zone center and are therefore optically inactive they cannot be probed by our $\mathrm{THz}$ techniques. We therefore focus our attention on the spin gap which relates to the spin anisotropy. The spin gap is directly measured by constant- $\mathbf{Q}$ energy scans at the zone center. Figure 6(b) shows such a scan at room temperature. A broad peak beginning at $\sim 2 \mathrm{meV}$ and falling off at 15 $\mathrm{meV}$ is attributed to magnetic scattering as the scan cuts through the spin-wave dispersion. The prominent peak at $10.3 \mathrm{meV}(2.37 \mathrm{THz})$ is attributed to the $\mathrm{Nd}^{3+}$ crystal field excitation [33]. As the sample is cooled below the SR to $3 \mathrm{~K}$ [Fig. 6(c)], the broad peak shifts to higher energies,

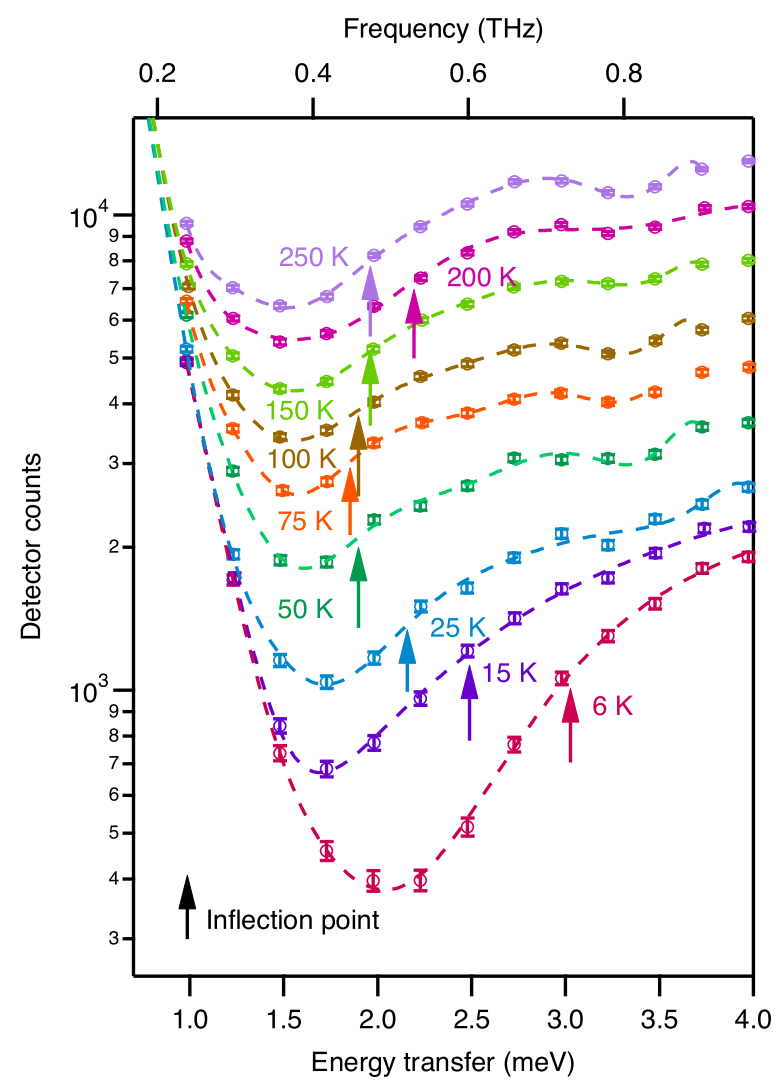

FIG. 7. (Color online) Constant $\mathbf{Q}$ energy scans of the scattering vector $\mathbf{Q}=(011)$ showing expansion of the spin gap with reducing temperature. Dashed lines represent polynomial fits. Arrows show inflection points of fits representing the spin-gap energies. 
now beginning at $\sim 3.75 \mathrm{meV}$. This is consistent with the development of the spin gap in Fig. 6. A background scan taken at $3 \mathrm{~K}$ with $\mathbf{Q}=\left(\begin{array}{lll}0.9 & 0 & 0.9\end{array}\right)$ reveals the non-spinwave contributions. Subtraction of the background gives zero readings below the spin-gap energy. This demonstrates both the magnetic nature of the features at the zone center and that the intensity across the spin gap is consistent with background levels. The stiff dispersion and resilience of the magnetic scattering over the broad temperature range up to $300 \mathrm{~K}$ discounts potential electromagnon-type excitations in regard to the $\mathrm{THz}$ measurements.
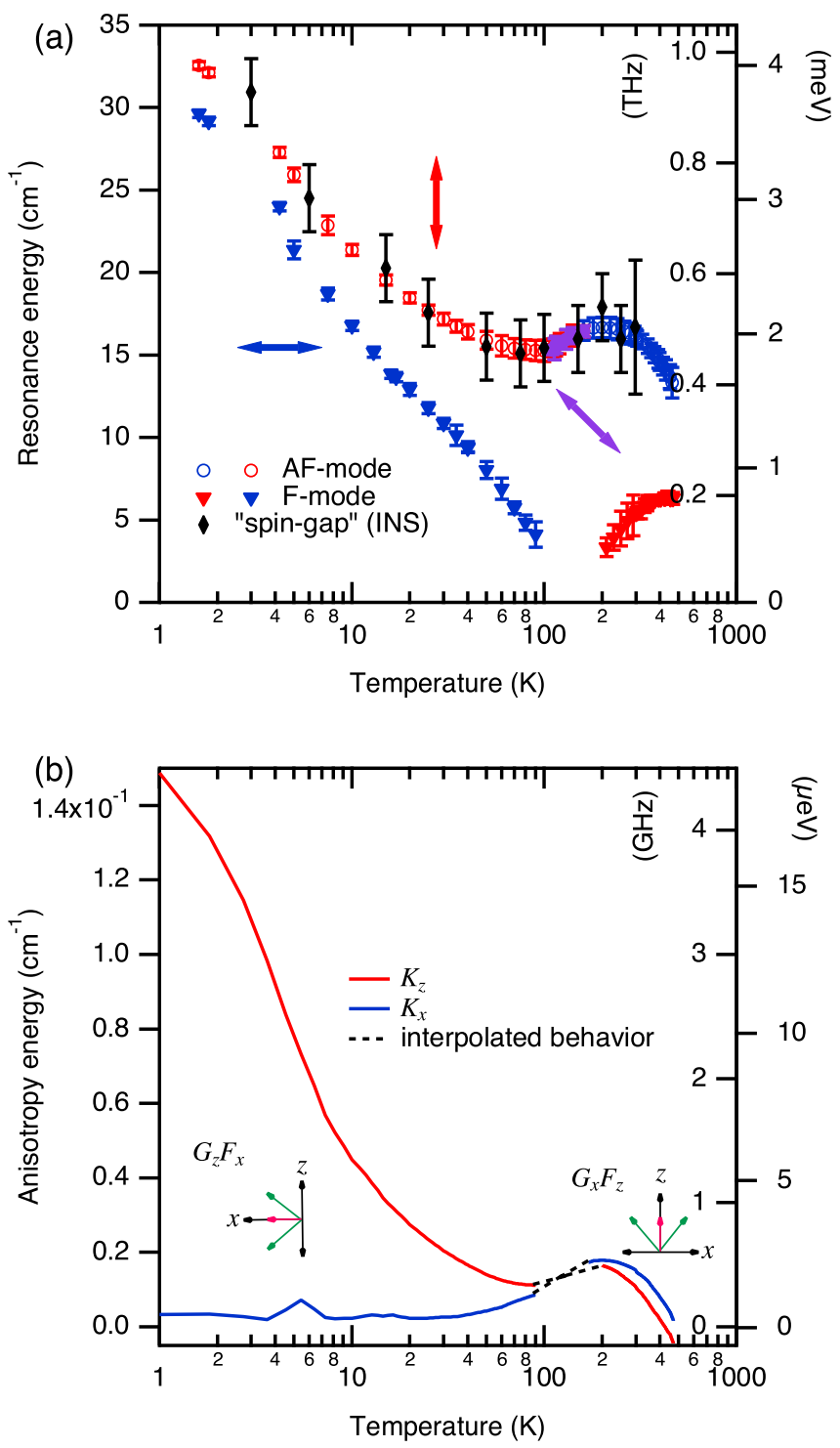

FIG. 8. (Color online) (a) Temperature dependence of F- and AF-mode energies with uncertainties representing half width at half maximum of fitted peaks. The temperature dependence of the spin gap from the INS results is overlaid. The uncertainties represent the resolution of the data points in the INS spectra. (b) Calculated temperature dependence of the effective anisotropy constants. The dashed line represents the predicted trend as the F mode completely softens over these temperatures and solutions to Eqs. (2) and (3) are unavailable.
Figure 7 displays a series of the temperature-dependent measurements for the spin gap attributed to the anisotropylifted spin waves. Here, the constant $\mathbf{Q}$ scattering spectra are taken at the $\mathbf{Q}=(011)$ scattering vector. Shapiro et al. [22] have shown in other $\mathrm{RFeO}_{3}$ compounds that spin waves in the $(0 \mathrm{kl})$ scattering plane feature an identical frequency dependence on temperature behavior to the $(h 0 l)$ scattering plane shown in Fig. 6. The spin gap is determined by a polynomial fit to the scattering data points. The zero-crossing point of a second-order differential gives the inflection point. The shift in the inflection point with reduced temperature is attributed to the changing magnetic anisotropy. When compared to the frequency of the AF mode as determined by $\mathrm{THz}$ spectroscopy, the inflection point energy shows striking agreement [Fig. 8(a)].

\section{Anisotropy calculation}

The temperature dependence, from 1.6 to $470 \mathrm{~K}$, of both modes observed using $\mathrm{THz}$ optical techniques is given in Fig. 8(a). The spin-gap energy, taken from the INS experiments, is overlaid. The spin-gap energies consistently match the energies of the AF-mode resonance. The lower-energy $\mathrm{F}$ mode, observed in our optical measurements, is inaccessible to the neutrons at temperatures above the SR, being obscured by the elastic Bragg peak. At low temperatures the F and AF modes have similar energies and the weak nature of the F mode and limited resolution of the triple-axis spectrometer explain why it is not resolved in the INS spectra.

We now present the first calculations for the anisotropy energies along the $x$ and $z$ magnetic axes as a function of temperature, by using Eqs. (2) and (3) and the data of Fig. 8(a). Values for $J, D$, and $\beta$ (given in Sec. II A) are from Ref. [29] and are assumed to change little over the temperature range considered here [20]. We take into account that the anisotropic field acting on the AF mode switches from $K_{x}$ to $K_{z}$ during the SR transition. At the same time, $\left(K_{x}-K_{z}\right)$, acting on the $\mathrm{F}$ mode, switches sign. The calculation could not be carried out from 90 to $210 \mathrm{~K}$ for the $\mathrm{F}$ mode as the optical data required are not available. The values shown in Fig. 8(b) represent the effective anisotropies along the $a$ and $c$ crystal axes. The result strongly suggests that the change in size of $K_{x}$ over $K_{z}$ (favoring antiferromagnetic alignment along $x$ over $z$ ) is the effective driving mechanism of the SR.

A complete assessment of the microscopic anisotropy contributions requires separate treatment of each term in the Hamiltonian. In reality, there may be numerous sources for anisotropic effects at the microscopic level. Some terms typically considered important in the literature involve secondand fourth-order single ion anisotropies, as well as dipoledipole spin interactions, giving an anisotropic contribution to the Hamiltonian of the form [14]

$$
H_{A}=\sum_{i, j}\left(\mathbf{K}_{2} \cdot \mathbf{S}_{i}\right)^{2}+\sum_{i, j}\left(\mathbf{K}_{4} \cdot \mathbf{S}_{i}\right)^{4}+\sum_{\substack{i, j \\ i \neq j}}\left(\mathbf{K}_{\mathrm{DP}} \cdot \mathbf{S}_{i}\right)^{2}
$$

Here $K_{2}$ and $K_{4}$ are the second- and fourth-order single ion anisotropies, and $K_{\mathrm{DP}}$ is the effective dipole-dipole interaction anisotropy. With $K_{2} \gg K_{4}$, and $K_{4}$ usually considered temperature independent $[13,14]$, containing the temperature dependence within an effective second-order anisotropy is a 
good approximation. Competition between the internal microscopic anisotropy forces equates to changes in the effective anisotropies along the $x$ and $z$ directions. For example, calculations performed by Bidaux et al. [34] show that the dipoledipole interactions for the $\mathrm{Fe}-\mathrm{Fe}$ sub-lattice in $\mathrm{RFeO}_{3}$ strongly favor an $x$ direction of antiferromagnetism as seen in $\mathrm{NdFeO}_{3}$ above the SR. Therefore, a considerable contribution to the temperature dependence of our experimentally determined $K_{x}$ value is likely to be from the dipole-dipole interactions within the Fe-Fe sublattice. At the same time, upon cooling, reduced thermal interactions may allow polarization of the $\mathrm{Nd}^{3+}$ ions with the weak ferromagnetic moment of the Fe-Fe sublattice as supported by recent magnetometry results [31,35]. This form of $\mathrm{R}-\mathrm{Fe}$ coupling may drive a modulation of the spin-lattice coupling, which in turn facilitates the onset of a new anisotropy along the $z$ direction. The rapid increase of our experimentally determined $K_{z}$ value should contain the information about this net effect. It is therefore conceivable that with our measurements one could subtract theoretically modeled sources of anisotropy, thus revealing the contributions of presently unknown interactions to better understand the underlying mechanism of SR.

\section{CONCLUSION}

In conclusion, we have shown that polarized $\mathrm{THz}$ waves are effective probes of the magnetic interactions in antiferromagnets with the ability to map the dynamic anisotropy, to high precision, across a broad temperature range. The combined use of continuous $\mathrm{THz}$ absorption techniques (Fourier transform infrared, photomixer source) and an ultrafast pulsed $\mathrm{THz}$ source (TDS) gave consonant results over the wide temperature range. Inelastic neutron scattering at the magnetic zone center $\mathbf{Q}=(101)$ confirms the magnetic origin of features evident in the $\mathrm{THz}$ spectra and provides evidence for a spin gap of the acoustic spin waves. A correlation between the spin gap in the spin-wave dispersion and the optically detected spin-wave modes in $\mathrm{NdFeO}_{3}$ provides a link between the SR and the variable magnetic anisotropy with final calculations showing that the SR transition occurs when $K_{x}$ is overcome by $K_{z}$. The ability to understand and tune the infrared absorption in the rare-earth orthoferrites not only adds to the development of the physics associated with spintronic and photonic functionality but also offers the potential for device applications such as optically-active filters of $\mathrm{THz}$ frequency light.

\section{ACKNOWLEDGMENTS}

We acknowledge the support of the Bragg Institute, Australian Nuclear Science and Technology Organisation, in providing the neutron research facilities used in this work. Travel funding for the neutron experiments was provided by the Australian Institute of Nuclear Science and Engineering. This work is also supported by the National Natural Science Foundation of China (NSFC, Grants No. 50932003 and No. 51372149) and by the Australian Research Council.
[1] M. R. Freeman and Z. Diao, Nat. Photonics 6, 643 (2012).

[2] T. Kampfrath, A. Sell, G. Klatt, A. Pashkin, S. Mährlein, T. Dekorsy, M. Wolf, M. Fiebig, A. Leitenstorfer, and R. Huber, Nat. Photonics 5, 31 (2011).

[3] A. V. Kimel, B. A. Ivanov, R. V. Pisarev, P. A. Usachev, A. Kirilyuk, and T. Rasing, Nat. Physics 5, 727 (2009).

[4] S. Wienholdt, D. Hinzke, and U. Nowak, Phys. Rev. Lett. 108, 247207 (2012).

[5] S. Mangin, D. Ravelosona, J. A. Katine, M. J. Carey, B. D. Terris, and E. E. Fullerton, Nat. Mater. 5, 210 (2006).

[6] J. Jeong, E. A. Goremychkin, T. Guidi, K. Nakajima, G. S. Jeon, S.-A. Kim, S. Furukawa, Y. B. Kim, S. Lee, V. Kiryukhin, S.-W. Cheong, and J.-G. Park, Phys. Rev. Lett. 108, 077202 (2012).

[7] M. Matsuda, R. S. Fishman, T. Hong, C. H. Lee, T. Ushiyama, Y. Yanagisawa, Y. Tomioka, and T. Ito, Phys. Rev. Lett. 109, 067205 (2012).

[8] C. Ederer and N. A. Spaldin, Phys. Rev. B 71, 060401(R) (2005).

[9] M. Nakajima, A. Namai, S. Ohkoshi, and T. Suemoto, Opt. Express 18, 18260 (2010).

[10] K. Yamaguchi, M. Nakajima, and T. Suemoto, Phys. Rev. Lett. 105, 237201 (2010)

[11] K. Yamaguchi, T. Kurihara, Y. Minami, M. Nakajima, and T. Suemoto, Phys. Rev. Lett. 110, 137204 (2013).

[12] A. Pimenov, A. A. Mukhin, V. Y. Ivanov, V. D. Travkin, A. M. Balbashov, and A. Loidl, Nat. Phys. 2, 97 (2006).

[13] J. R. Shane, Phys. Rev. Lett. 20, 728 (1968).

[14] L. M. Levinson, M. Luban, and S. Shirikman, Phys. Rev. 187, 715 (1969).
[15] T. Yamaguchi, J. Phys. Chem. Solids 35, 479 (1974).

[16] W. C. Koehler, E. O. Wollan, and M. K. Wilkinson, Phys. Rev. 118, 58 (1960).

[17] R. L. White, J. Appl. Phys. 40, 1061 (1969).

[18] D. Treves, Phys. Rev. 125, 1843 (1962).

[19] E. F. Bertaut, J. Appl. Phys. 33, 1138 (1962).

[20] W. Sławiñski, R. Przeniosło, I. Sosnowska, and E. Suard, J. Phys.: Condens. Matter 17, 4605 (2005).

[21] R. W. Grant and S. Geller, Solid State Commun. 7, 1291 (1969).

[22] S. M. Shapiro, J. D. Axe, and J. P. Remeika, Phys. Rev. B 10, 2014 (1974).

[23] G. F. Herrmann, J. Phys. Chem. Solids 24, 597 (1963).

[24] R. M. White, R. J. Nemanich, and C. Herring, Phys. Rev. B 25, 1822 (1982).

[25] D. Treves, J. Appl. Phys. 36, 1033 (1965).

[26] S. Yuan, Y. Wang, M. Shao, F. Chang, B. Kang, Y. Isikawa, and S. Cao, J. Appl. Phys. 109, 07E141 (2011).

[27] E. Constable, J. Horvat, D. L. Cortie, Z. Cheng, S. Yuan, S. Cao, and R. A. Lewis, 38th International Conference on Infrared, Millimeter, and Terahertz Waves (IRMMW-THz), (IEEE, Mainz, 2013), pp. 1-2.

[28] E. Constable and R. A. Lewis, J. Appl. Phys. 112, 063104 (2012).

[29] N. Koshizuka and K. Hayashi, J. Phys. Soc. Jpn. 57, 4418 (1988).

[30] R. Przeniosło, I. Sosnowska, and P. Fischer, J. Magn. Magn. Mater. 140-144, 2153 (1995).

[31] S. J. Yuan, W. Ren, F. Hong, Y. B. Wang, J. C. Zhang, L. Bellaiche, S. X. Cao, and G. Cao, Phys. Rev. B 87, 184405 (2013). 
[32] L. M. Helme, A. T. Boothroyd, R. Coldea, D. Prabhakaran, A. Stunault, G. J. McIntyre, and N. Kernavanois, Phys. Rev. B 73, 054405 (2006).

[33] R. Przeniosło, I. Sosnowska, M. Loewenhaupt, and A. Taylor, J. Magn. Magn. Mater. 140-144, 2151 (1995).
[34] R. Bidaux, J. E. Bouree, and J. Hammann, J. Phys. Chem. Solids 35, 1645 (1974).

[35] Y. B. Bazaliy, L. T. Tsymbal, G. N. Kakazei, and P. E. Wigen, J. Appl. Phys. 95, 6622 (2004). 